LONDOÑO, Fernando, "Recensión: American Criminal Law Review, Vol. 45 (2008), n 3 y 4" Polit. crim. No 7, 2009, R2-7, pp. 4-8.

\title{
Recensión: American Criminal Law Review, Vol. 45 (2008), fascículos 3 y 4
}

\author{
Fernando Londoño M. \\ Dr. en Derecho, Centro de Estudios de Derecho Penal de la Universidad de Talca \\ flondono@utalca.cl
}

Continuando con la recensión del volumen 45 de la ACLR, iniciada en el número pasado de esta revista, toca dar cuenta de los fascículos de clausura, correspondientes a los trimestres de verano y otoño del hemisferio boreal. Los ejemplares incluyen un total de seis artículos y seis notas o comentarios de jurisprudencia. Los artículos ofrecen una completa gama de temas: teoría general de la norma penal y de su aplicación; ejecución de la pena de muerte y bioética; criminología desde una perspectiva económica neoclásica; proceso penal y responsabilidad de las personas jurídicas; política criminal en contextos institucionales y normativos complejos, como es el caso del mercado de valores.

El fascículo estival se abre con el trabajo de Kay L. Levine, The External Evolution of Criminal Law ("La evolución externa del derecho penal", fasc. 3, pp. 1039 - 1100). ¿Qué factores determinan la aplicación o interpretación de la normas penales y su mutación en el tiempo? ¿Qué circunstancias o fuerzas explican la diferencia - históricamente creciente entre la law on the books y la law in action en el campo penal? Estas preguntas son afrontadas por la autora a la luz del denominado modelo de evolución externa, aplicado al caso de la legislación californiana del históricamente maleable tipo penal de violación impropia. ${ }^{1}$ La originalidad del trabajo radica precisamente en el método de observación propuesto al efecto: el modelo de evolución externa busca explicaciones no tanto en las variables internas de evolución (factores institucionales y cambios desde los operadores del propio sistema penal), como en los factores y fuerzas sociales en acto (cambios socialesestructurales, opinión públicas y medios masivos de comunicación, entre otros).

A continuación, en How Lethal Injection Reform Constitutes Impermissible Research On Prisoners ("Sobre cómo la reforma en materia de inyecciones letales constituye una forma inadmisible de investigación en prisioneros", fasc. 3, pp. 1101 - 1166), Seema Shah aborda un aspecto descuidado en el debate seguido a continuación del caso Baze v. Rees, resuelto en 2008 por la Corte Suprema de los EE.UU.: ${ }^{2}$ a juicio de la autora, los estándares de

\footnotetext{
${ }^{1}$ La autora hace ver cómo, pese a que el tipo penal positivo se ha mantenido fundamentalmente inalterado en el tiempo, su significado o la comprensión de la lesividad causada por la conducta sexual de adultos contra menores de edad "ha sido reinventada muchas veces en los últimos 150 años, y no sólo en aspectos marginales, sino en su núcleo" (p. 1044). Así, el tipo penal de violación impropia ha sido utilizado sucesiva y alternativamente para "reforzar el interés de los padres en la posibilidades de las hijas de acceder al matrimonio, para proteger la castidad de las jóvenes contra hombres seductores, para controlar a mujeres adolescentes promiscuas o afectadas por enfermedades, para incrementar los financimientos de apoyo a los niños, para reducir los embarazos de adolescentes y para determinar y castigar la explotación sexual de los adolescentes" (p. 1044).

${ }^{2}$ Baze v. Rees, No. 07-5439 (U.S. Apr. 16, 2008), en el que, en un fallo muy dividido, la Corte Suprema de los EEUU. reconoció la constitucionalidad del método de inyección letal del estado de Kentucky, a la vez que
} 
CENTRO DE ESTUDIOS DE DERECHO PENAL. "Revista de Revistas."

Polit. crim. No 7, 2009, R2-7, pp. 1-18.

[http://www.politicacriminal.cl/n_7/r_2_7.pdf]

constitucionalidad de la inyección letal ofrecidos por la Corte Suprema no resultan para nada claros, lo que está llevando a los estados a promover reformas en materia de inyección letal, a tientas; de esta forma, en orden a cumplir con los estándares, los estados han ido experimentando con distintos procedimientos y protocolos, conduciendo así investigaciones legal y éticamente intolerables en prisioneros. Concretamente, los estados están variando las dosis y tipos de drogas usadas; están desarrollando métodos diseñados para que profesionales no médicos administren procedimientos médicos; y están recabando información para aprender de la experiencia de ejecuciones fallidas.

Con ello se pasa al tercer artículo del fascículo estival, único de carácter netamente criminológico. Durante los años 2005 y 2006 la tasa de los delitos de robo experimentó un claro repunte en los EE.UU. ¿Qué factores explican dicha alza? John K. Roman y Aaron Chalfin dan una interesante respuesta a esta pregunta en "¿Ha aumentado la demanda de crímenes? La relevancia de los artefactos mediales portátiles y el alza de los robos en 2005 y 2006" (Has Demand for Crime Increased? The Prevalence of Personal Media Devices and the Robbery Spike in 2005 and 2006, fasc. 3, pp. 1149 - 1166). Para ello, los autores hacen suyo el modelo criminológico de la teoría económica neoclásica, ${ }^{3}$ pero se concentran en dos de los factores menos atendidos por los seguidores de dicho modelo, de entre aquellos incidentes en la "oferta" de delitos; a saber: cambios en las ganancias esperadas de una transacción criminal (expected profits of a criminal transaction) y cambios en las precauciones o medidas preventivas adoptadas por los particulares. Pues bien, los autores observan que en 2005 y 2006 los consumidores estadounidenses adquirieron diez millones de artefactos mediales portátiles, particularmente iPods; y que ello no se acompañó de un incremento en el nivel de prevención privada del delito; antes bien, estos costosos artefactos fueron (y son) abiertamente expuestos en público, reduciendo con ello los "costos de búsqueda" en favor de los potenciales agresores. Las conclusiones son entonces evidentes: factores exógenos, como cambios significativos en los patrones de consumo y uso de bienes, sumado a reducciones en los niveles de prevención privada (todo lo cual incide fuertemente en el nivel de ganancias esperadas de una transacción criminal) podrían explicar el mencionado repunte de la tasa de delitos de robo.

Una muestra de lo articulada e intensa que puede ser la discusión procesal penal estadounidense en sede de responsabilidad de las personas jurídicas, está dada por el artículo de Julie R. O'Sullivan, que encabeza el fascículo 4 del volumen en comento y que podría traducirse como " ¿Acaso la política de renuncia al privilegio propiciada por el Departamento de Justicia pone en jaque el sentido implícito en el privilegio abogadocliente $^{4}$ y en la doctrina del work product?" Un "no" preliminar" (Does DOJ's Privilege

ofreció una serie de estándares para la aplicación de la Octava Enmienda sobre prohibición de castigo cruel, a la ejecución por inyección letal.

3 Conforme a la cual existe un "mercado para el delito", el que se comporta de manera muy similar a cualquier otro mercado. Su primer planteamiento detallado se debe a Gary S. Becker, Crime and Punishment: An Economic Approach, 76 J. POL. ECON. 169 (1968).

${ }^{4}$ En virtud del denominado attorney-client privilege, las comunicaciones entre el abogado y su cliente quedan protegidas bajo confidencialidad. En el ámbito de la responsabilidad de las empresas, un hito en este contexto está representado por la doctrina emanada a partir del caso Upjohn Co. v. United States (449 U.S. 383, 1981), en el que la Corte Suprema sostuvo que una persona jurídica puede invocar el privilegio en cuestión para 
LONDOÑO, Fernando, "Recensión: American Criminal Law Review, Vol. 45 (2008), n 3 y 4" Polit. crim. No 7, 2009, R2-7, pp. 4-8.

Waiver Policy Threaten the Rationales Underlying the Attorney-Client Privilege and Work Product Doctrine? A Preliminary "No", fasc. 4, pp. 1237 - 1296). El autor critica los recientes intentos legislativos en orden a impedir que los fiscales (Departamento de Justicia) soliciten a las personas jurídicas la renuncia de los privilegios en cuestión a cambio de beneficios procesales o penales para las mismas (con el simultáneo desmedro para los directivos o empleados imputados en el caso). El autor sostiene que con este tipo de legislación el Legislativo yerra el tiro, pues la política de renuncia de privilegios y negociación no sería más que una consecuencia del problema de fondo; a saber, el enorme poder fáctico de que está dotado el órgano persecutor en el marco de los procesos contra personas jurídicas en atención a: 1.- la inexistencia de una Quinta Enmienda a favor de las personas jurídicas; ${ }^{6}$ 2.- la amplitud de los estándares de responsabilidad de las personas jurídicas; 3.- la "elasticidad" de los preceptos del Código Penal Federal y 4.- el amplio espectro y dureza de las sanciones disponibles contra las personas jurídicas. En suma, el autor argumenta que los esfuerzos legislativos deberían concentrarse en la moderación de las circunstancias que dan poder al Departamento de Justicia y a sus fiscales, y no en la mera restricción de su uso o ejercicio.

Luego, en "Sobre el registro de las declaraciones de imputados detenidos por delitos federales" (Recording Federal Custodial Interviews, fasc. 4., pp. 1297 - 1345), Thomas P. Sullivan expone con todo detalle las razones que hacen aconsejable la adopción de grabaciones o registros electrónicos de las interrogaciones en cuestión. Dicha práctica es resistida por los órganos de prosecución de nivel federal (a diferencia de la tendencia a nivel estatal), quienes se limitan a elaborar reportes, fundamentalmente a partir de las notas tomadas por los funcionarios durante la declaración.

La serie de artículos se cierra con el artículo de Emil J. Bove III, "Los factores institucionales que sustentan las decisiones de acusación criminal en contextos regulatorios complejos" (Institutional Factors Bearing on Criminal Charging Decisions in Complex Regulatory Environments, fasc. 4, pp. 1347 - 1387). Se trata de un refinado artículo sobre política criminal en contextos institucionales y normativos complejos, como es el caso del mercado de valores. ¿Qué factores deben considerar los órganos persecutores al levantar cargos en un contexto semejante? ¿Qué dificultades cognitivas deben enfrentar los fiscales en un contexto técnica y normativamente muy complejo, y de cara a defensas análogamente complejas? ¿Y qué dificultades deben enfrentar para traducir esa complejidad de manera que sea comprensible y aceptables para los jurados? El autor aborda estos problemas exponiendo en primer lugar una serie de acusaciones sostenidas por fiscales desde 2005 a esta parte contra diversos actores del mercado bursátil (conflictos de intereses de market makers, fraudes o administraciones desleales de administradores de hedge funds, entre

\footnotetext{
proteger no sólo las comunicaciones entre los abogados y los gerentes y directivos, sino también aquellas entre el abogado y otro tipo de empleados no directivos.

${ }^{5}$ En los Estado Unidos, la denominada working paper doctrine protege el carácter reservado del material preparado por una parte con miras al juicio, de manera que la contraparte queda impedida de solicitar su exhibición o producción.

${ }^{6}$ Corresponde a una garantía constitucional que en general tutela a los ciudadanos contra abusos de la autoridad gubernamental en el contexto de un procedimiento legal; incluye el derecho al gran jurado en casos de crímenes mayores, el non bis in idem y el nemo tenetur, entre otros.
} 
CENTRO DE ESTUDIOS DE DERECHO PENAL. "Revista de Revistas."

Polit. crim. No 7, 2009, R2-7, pp. 1-18.

[http://www.politicacriminal.cl/n_7/r_2_7.pdf]

otros), pero que en definitiva no prosperaron como se esperaba. Precisamente a propósito de estos casos, el autor ilustra los desafíos que típicamente debe afrontar un fiscal en estos ámbitos, a la vez que ofrece perspectivas de respuesta. A este propósito, Emil Bove anuncia su mayor preocupación, la que por lo demás es muy propia de la sensibilidad jurídica anglosajona: la atención a los efectos de las puntuales decisiones de persecución penal en el conjunto del sistema; es decir, la consideración de las externalidades de una decisión. Asumiendo que en algunos de estos casos las decisiones de los fiscales pudieron impactar negativamente en el sistema, el autor hace una llamado a la necesidad de una muy cuidadosa ponderación de los eventuales efectos de las formulaciones de cargo, especialmente por lo que se refiere al impacto en las sedes legislativas y administrativas de regulación, así como para los actores institucionales en general (jurados, tribunales, etc.).

Los restantes seis trabajos de los ejemplares aquí reseñados, corresponden exclusivamente a notas o comentarios de jurisprudencia estadounidense. En la nota intitulada Effective Warnings Before Consent Searches: Practical, Necessary, and Desirable ("Advertencias efectivas antes de los registros consentidos: prácticas, necesarias y deseables", fasc. 3 pp. 1185 - 1212), Matthew Phillips expone su rechazo a la doctrina procesal penal sentada por la Corte Suprema de los EE.UU. en Schneckloth v. Bustamonte, ${ }^{7}$ según la cual resulta inviable para el Estado requerir una advertencia efectiva y detallada del derecho a refutar una solicitud normal de registro voluntaria o consentida (consent searches). El autor argumenta que el requerimiento de una advertencia efectiva de derechos es necesario para paliar la coerción inherente a los registros consentidos y para reducir el uso racialmente discriminatorio de los mismos. Los volúmenes en comento incluyen otros cinco comentarios de jurisprudencia sobre argumentos diversos y de alcance principalmente local; ${ }^{8}$ a saber, "Jamás eficiente, pero siempre libre: sobre por qué la Juvenile Adjudication Question $^{9}$ representa el último signo de que la jurisprudencia de Almendarez-Torres $v$. United States ${ }^{10}$ debe ser revocada", de Molly Gulland (fasc. 3, pp. 1167-1184); "Reviviendo la "Law Office History": sobre cómo las fuentes históricas y académicas influencian a la jurisprudencia de la Segunda Enmienda", de Adam Small (fasc. 3. pp. 1213-1235); "Un examen empírico de los factores asociados a las conmutaciones de condenas de muerte entre 1986 y 2005", de John Kraemer (fasc. 4, pp. 1389 -1417); “¿Cómo aplican las Cortes Federales de Apelación la revisión de razonabilidad de Booker, después de Gall?", ${ }^{11}$ de Anne Louise Marshall (fasc. 4, pp. 1418 - 1438 ). Por último,

\footnotetext{
${ }^{7} 412$ U.S. 218, 231 (1973).

${ }^{8}$ Véase en todo caso la nota final, que cierra el volumen de 2008: ¡lamentablemente no hay nada de local en ella!

${ }^{9}$ Véase la nota siguiente.

${ }^{10}$ En el caso Almendarez-Torres v. United States de 1998 (523 U.S. 224 - 1998), la Corte Suprema de los EE.UU. decidió que toca al juez y no al jurado, decidir si una condena precedente tiene o no efectos en sede de agravación de pena. Esta doctrina aparece como una excepción en el marco de los derechos de la Sexta Enmienda y desde su pronunciamiento han habido sucesivos intentos en orden a restringir su alcance. Entre ellos, se cuenta la idea de excluir de dicha doctrina las adjudicaciones precedentes en calidad de adolescente, no pronunciadas por jurados. En tal sentido se pronuncia la autora de la nota.

${ }^{11}$ El título alude a la jurisprudencia emanada de la Corte Suprema de los EEUU. a partir de los casos US. $v$. Booker y Gall v. US. La discusión atañe a los estándares de revisión utilizados en sede de apelación, en los casos en los que la sentencia de primer grado se ha apartado - razonablemente o no -de las Sentencing Guidelines.
} 
LONDOÑO, Fernando, "Recensión: American Criminal Law Review, Vol. 45 (2008), n 3 y 4" Polit. crim. No 7, 2009, R2-7, pp. 4-8.

cierra el volumen 45 de 2008 la nota "Hipotecas y delitos menores: el enforcement penal de los requerimientos de la licencia para préstamos hipotecarios estatales y protección de los propietarios de habitaciones", de Christopher Steelman (fasc. 4, pp. 1439 - 1459). Un cierre de año simbólico y terrenal, cual si la ACLR quisiera recolocar nuestra atención en la concreta y áspera materia de la actual crisis económica mundial, la que, como es sabido, comenzó precisamente con un "banal" problema de créditos hipotecarios en los EE.UU. E1 propio autor de la nota resume dicho origen valiéndose del inigualable american style: "the proverbial "popping" of the "housing bubble." (p. 1439). 